\title{
La información como recurso estratégico en las empresas de base tecnológica
}

\author{
Ivett M. APORTELA RODRIGUEZ \\ Universidad Carlos III de Madrid. \\ Facultad de Humanidades, Comunicación y Documentación. \\ Departamento de Biblioteconomía y Documentación \\ iaportel@bib.uc3m.es \\ Cristina GALLEgo GóMEZ \\ Capgemini \\ cristina.gallegogo@gmail.com
}

Recibido: Junio 2015

Aceptado: Noviembre 2015

\begin{abstract}
Resumen:La información es un recurso estratégico para las empresas de base tecnológica. Pero, su valor no suele estar reconocido, y por ende, no suele potenciarse ni gestionarse eficazmente, poniendo en peligro el desenvolvimiento y la supervivencia de dichas organizaciones. Es por ello que el presente artículo hace un análisis de la percepción de la información como recurso estratégico para el desarrollo de estas empresas, a partir de la literatura especializada; en lo que constituye un primer acercamiento al tema. Se ofrece una síntesis de los principales hallazgos en la revisión bibliográfica y se desarrolla el estado del arte sobre el tema. Se caracterizan las empresas de base tecnológica y la información en la empresa. Posteriormente se describe el impacto de la información en las EBTs y el rol de los parques científicos y tecnológicos como mediadores en el uso de la información.
\end{abstract}

Palabras clave: Conocimiento; Empresas de base tecnológica; Gestión de la información; Información; Inteligencia competitiva.

\section{Information as a strategic resource intechnology-based companies}

\begin{abstract}
Information is a strategic resource in technology-based companies. But often its value is not recognized, meaning it is not enhanced nor managed effectively, endangering the development and even survival of these organizations. This article analyzes the descriptions in the literature of how such companies view information as a strategic resource, to provide an initial approach to the topic. A summary of the most important results, and a broad view of the state of the art are given. Technology-based companies and information used in them is characterized, the impact of information in these companies is described, and the role of Science and Technology Parks as mediators in information use is analyzed.
\end{abstract}

Keywords: Competitive intelligence; Information; Information management; Knowledge; Technology-based companies. 


\section{INTRODUCCIÓN}

La informaciónen la sociedad actualse ha convertido en un bien de consumo que existe la necesidad de gestionar para garantizar la explotación de los datos y el conocimiento de la empresa. Su uso eficiente permite la mejora de los flujos informacionales, y optimiza los procesos organizacionales.

Como activo intangible la información está presente en todas las organizaciones y constituye un componente 'invisible' indispensable para hacer 'visible' el resto de los recursos. Esla principal fuente de conocimientos de la empresa; de ahí, que sea la base de los procesos de investigación, desarrollo e innovación tecnológica $(\mathrm{I}+\mathrm{D}+\mathrm{i})$; y un elemento clave para lograr alcanzar altos niveles de competitividad empresarial. Un hecho que es corroborado por Pérez González y Placer Maruri (2011: 496), quienes reconocen que "la competitividad de las organizaciones se ve comprometida por su capacidad de reacción y adaptación al entorno, mediante una adecuada gestión de la información y el conocimiento que dé lugar a nuevos productos y a procesos más eficientes”.

En el caso de las empresas de base tecnológica [EBTs], caracterizadas por ser intensivas en conocimiento e innovación, la información adquiere un rol estratégico que no siempre es reconocido. Por una parte, los procesos de toma de decisiones muchas veces se realizan en base a sentimientos, intuiciones y a un análisis superficial de las perspectivas de mercado, raramente basados en informaciones adecuadas sobre la actividad de la empresa (Netto, 2006: 6). Mientras que por la otra, la información no siempre se gestiona de forma efectiva, lo que redunda en un menor beneficio organizacional.

La gestión de información, en especial la del entorno, se constituye por tanto, como un proceso fundamental en las EBTs; de ahí que, sea necesario planificarlo y ejecutarlo, en correspondencia con los objetivos de la organización. Sin embargo, esta premisa no llega a ser una cuestión plenamente asimilada por los emprendedores, quienes no siempre reconocen su necesidad.

Teniendo en cuenta esta realidad,el presente artículotiene como objetivo hacer un análisis de la percepción de la informacióncomo recurso estratégico para el desarrollo de las EBTs, a partir de la literatura especializada. En un primer momento, seresumen los datos más significativos de la revisión bibliográfica realizada y a continuación se describe el estado del arte sobre el tema. Seesbozan las características más representativas de este tipo de organizaciones y sedescribe el comportamiento de la información en las empresas, haciendo énfasis en su valor como fuente de conocimiento y en la importancia de la utilización de la vigilancia tecnológica y la inteligencia competitiva como herramientas para la gestión de la información del entorno. Por último, se explica el impacto que puede tenerla información y su adecuada gestión en el funcionamiento de las EBTs, y cómo los parques científicos y tecnológicos y/o viveros de empresa, como impulsores de dichos proyectos empresariales, pueden incidir en el uso de este recurso. 


\section{METODOLOGÍA}

La revisión bibliográfica realizada pertenece a la fase exploratoria de una investigación más amplia que actualmente se encuentra en curso (Aportela Rodríguez, 2014)y que incluye entre otros elementos, un análisis del papel de la información en las EBTs a partir de la visión de los propios emprendedores y del rol que juegan los parques científicos y tecnológicos en este contexto. Todo ello con el objetivo de proponer soluciones que permitan apoyar a las empresas incubadas en sus procesos de gestión de la información.

Las principales fuentes de información empleadasen la búsqueda de la bibliografía han sido las bases de datos especializadas (ABI/INFORM Complete de ProQuest; Academic Search Premier y Library, Information Science \& Technology Abstract sde EBSCO; la Web of Science de Thomson Reuters, etc.) y los portales como DIALNET, SciELO y el Portal da CAPES (Brasil). El buscador de Google y Google Scholar también fueron empleados.

En cuanto a los criterios aplicados en la búsqueda y selección de la literatura especializada se han empleado palabras claves como: "empresa de base tecnológica” 'EBTS', “viveros de empresas”combinadas con términos como 'información', “inteligencia competitiva” o "gestión de información” entre otras. En inglés, por su parte se utilizaron "technology-basedcompany" o "technology based firm" unidos a "information management” o 'information'. Siempre que fue posible, las búsquedas estuvieron limitadas al título, la materia o el resumen; aunque en el caso de la Web of Science, se realizaron a partir de la opción 'Tema'. Se excluyeron los servicios de prensa, los periódicos y las revistas generales.

Dado que el objetivo del estudio no incluía hacer un análisis de la producción científica de los autores sino elaborar un estado del arte sobre el tema, se analizaron los documentos recopilados procedentes de las distintas fuentes y se excluyeron aquellos que no eran relevantes para la investigación, teniendo en cuenta su adecuación al tema y su capacidad para sentar bases conceptuales. Al respecto, debe señalarse que muchos de los estudios identificados estaban más enfocados al análisis de la información desde la perspectiva contable-financiera, de inversión, financiación, etc. que desde su propio valor como recurso estratégico.

\section{SÍNTESIS DE LA REVISIÓN BIBLIOGRÁFICA}

Como resultado de la revisión bibliográfica se identificaron un total de 62 documentos escritos en castellano, inglés y portugués; de los cuales, el 62,9\% correspondían a artículos de revista y actas de congreso, mientras que un 24,2\% eran libros y monografías. El resto estaba integrado por sitios web, informes de trabajo y trabajos de fin de curso.

El 33,9\% de los documentos han sido publicados en los últimos cuatro años, tal y como puede apreciarse en el gráfico 1. No obstante, para establecer el estado del 
arte sobre el tema, por lo general, han sido útiles documentos con una antigüedad mayor a dos años, puesto que los más recientes abordaban estudios de casos concretos con mayor correspondencia con la segunda fase de la investigación.

Gráfico 1. Distribución de los documentos por fecha de publicación

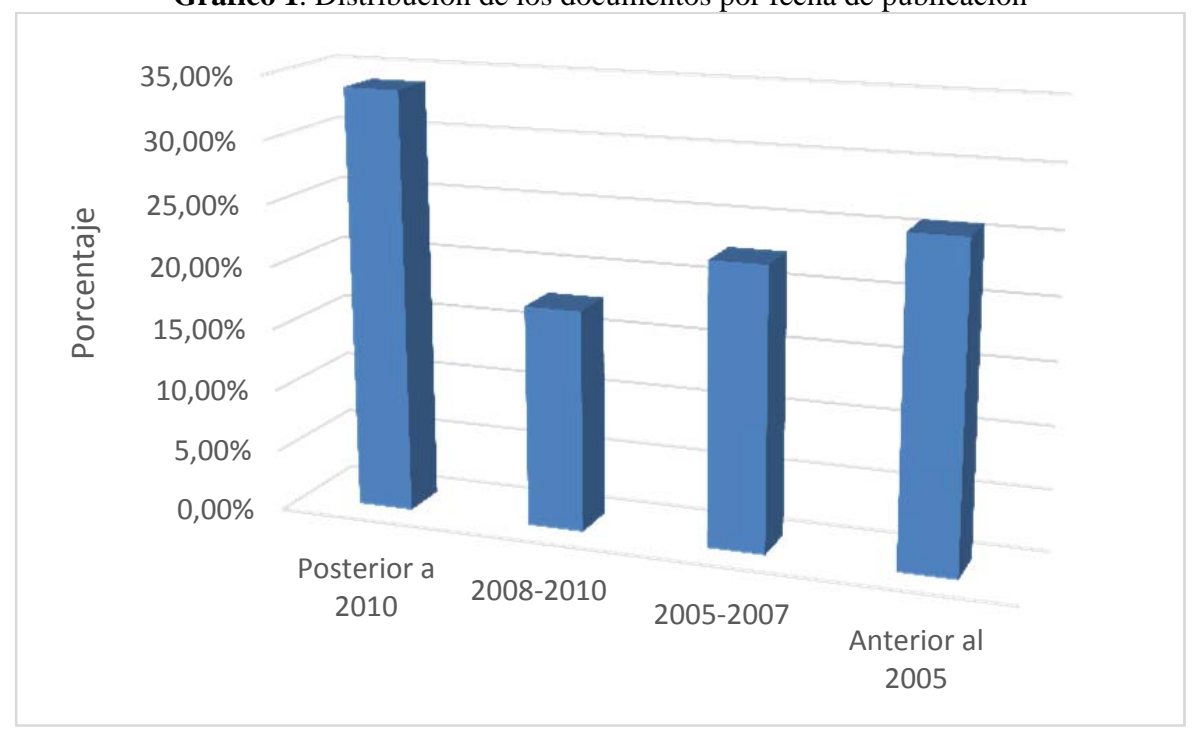

De los documentos localizados, autores como Netto (2006) o Feliú Rey (2011)han servido de base, desde la perspectiva general, para conocer las características de lasempresas de base tecnológica. Mientras que, Pablo Herederos (2012), en materia de sistemas de información o Escorsa Castell (2007) desde la inteligencia competitiva y la vigilancia tecnológica han ayudado a describir el rol de la información en la empresa.

Sin embargo, son pocos los estudios identificados que analizan la gestión de la información en las empresas de base tecnológica, lo que avala la necesidad de una investigación como la que se desarrolla. Esta problemática también ha sido señalada por Lofsten (2014 p. 247), cuya investigación busca establecer la relación entre las estructuras de información y la innovación.

La mayoría de las investigaciones sobre el papel de la información como recurso estratégico en dichas empresas, han sido realizadas en Brasil. Entre ellas, deben mencionarse la desarrollada por Gomes y Rocha (2011) con las empresas insertadas en un programa de incubación de la universidad Federal de Goiás (Brasil) y por Periotto(2010)asociada al uso de la información en las EBTs del Parque tecnológico de Sao Carlos(Brasil).

Desde la perspectiva de la inteligencia competitiva, también se pudieron identificar algunos artículos que han ayudado a perfilar el impacto de la información en las EBTs. Entre ellos, puede mencionarse el del profesor 
españolMerino Moreno (2011) que analiza la inteligencia competitiva en el entorno de las EBTs, como parte de la labor de gestión de la información externa que deben llevar a cabo estas empresas o los de Tanev (2007; Tanev y Bailetti, 2008), que estudian la inteligencia competitiva asociada a la innovación en las EBTs en el contexto canadiense.

\section{PAPEL DE LA INFORMACIÓN EN LAS EMPRESAS DE BASE TECNOLÓGICA: ESTADO DEL ARTE}

Las características intrínsecas de las empresas de base tecnológica hacen que en ellas la información como recurso estratégico adquiera dimensiones particulares. Por esta razón, resulta indispensable comenzar el análisis haciendo una descripción de este tipo de organizaciones para posteriormente aproximarnos al papel que juega la información en ellas.

\subsection{LAS EMPRESAS DE BASE TECNOLÓGICA: DEFINICIÓN Y PRINCIPALES CARACTERÍSTICAS}

Desde el punto de vista teórico, es posible analizar las empresas de base tecnológica (EBTs) partiendo del concepto de empresa. Al respecto, el Diccionario deReal Academia Española(2001)entiende la "unidad de organización dedicada a actividades industriales, mercantiles o de prestación de servicios con fines lucrativos". Dicho concepto ofrece una aproximación genérica, pero carece de matices que permitan reconocer las características propias de este tipo de organizaciones.

Para Storey y Tether (citados por Nieto y Santamaría, 2010: 1) las EBTs son entidades que tratan de desarrollar y explotar comercialmente una innovación tecnológica que implica una elevada incertidumbre. En esta misma línea, la Office of Technology Assessment, citada por varios autores (Feliú Rey, 2011: 9; Palacios, Val y Casanueva, 2005: 12, entre otros), las ha descrito como "organizaciones productoras de bienes y servicios, comprometidas con el diseño, desarrollo y producción de nuevos productos y/o procesos de fabricación innovadores, a través de la aplicación sistemática de conocimientos técnicos y científicos”.

El paralelismo entre el concepto general y el particular, incorpora términos que constituyen características propias de las EBTs, entre los que resulta más significativo el desarrollo de procesos técnicos a partir de la aplicación del conocimiento. Un hecho que implica un comportamiento muy diferente al de empresas similares en industrias tradicionales.

El desarrollo de estos proyectos empresariales y su consolidación como empresa de éxito no está exento de dificultades, sino todo lo contrario. Los emprendedores se enfrentan a su desconocimiento en materias gerenciales o de administración, pero también a las limitaciones de recursos económicos y financieros. A menudo carecen de capacidades técnicas y de marketing, sufren de 
mala gestión, incapacidad para encontrar financiación inicial y/o de altos gastos generales (Hackett y Dilts citados por Somsuk, Wonglimpiyarat y Wonglimpiyarat, 2012: 246). Ante estos problemas, el establecimiento de acuerdos de colaboración constituye una estrategia útil para lograr la competitividad necesaria. En este sentido, Nieto y Santamaría(2010: 4)hacen ver que las alianzas con las universidades y otros centros de investigación ayudan a las EBTs a estar en la vanguardia de los nuevos conocimientos y tecnologías.

En aras de contrarrestar las debilidades típicas de este tipo de organizaciones y con el propósito de conseguir el apoyo que necesitan en los primeros momentos, las EBTs muchas veces se insertan en ambientes dedicados a potenciar la relación entre la ciencia, la tecnología y la empresa. Un ejemplo de ellos son los parques científicos y tecnológicos, o en su defecto los viveros de empresa que pueden o no estar asociados a los anteriores. Estas instituciones buscan estimular la creación y el crecimiento económico a partir del conocimiento; al mismo tiempo que promueven activamente la transferencia de tecnología desde la academia y los centros de investigación al tejido empresarial.

Dentro de los parques científicos y tecnológicos, los viveros de empresa suelen ser la estructura encargada de fomentar, potenciar y apoyar los proyectos con un alto grado de innovación que inician su andadura empresarial. También pueden encontrarse fuera de estos, siendo la infraestructura que establece el nexo de la universidad con el mercado y/o la base para el posterior desarrollo de un parque, pero siempre con un propósito similar. Para Castillo Hermosa y Díez Fuente (2006: 142) son el "lugar en el que se concentran nuevos negocios en un espacio limitado con el objetivo de mejorar sus posibilidades de crecimiento y sus oportunidades de supervivencia”.

\subsection{LA INFORMACIÓN EN LA EMPRESA}

La información, aplicada al contexto propio de la empresa, se entiende como "todo aquello que sirve para poner de manifiesto la situación del entorno o del sistema, sus objetivos o resultados y reducir la incertidumbre frente a un proceso de decisión" (Pablos Heredero et al., 2012: 28).

A partir de la definición anterior se evidencia la importancia de contar con información sobre el entorno, es decir, conocer qué hacen los competidores, cuál es la normativa que rige la actividad de la empresa, cuál es la situación económica global, etc. Pero también resulta necesario mirar hacia el interior, saber cómo funcionan los flujos organizacionales, de qué forma los procesos informacionales apoyan a los fundamentales y cómo se transfiere e intercambia el conocimiento entre los trabajadores, etc. Este interés da pie a mirar la organización desde todas sus perspectivas, para tener identificadas las posibles oportunidades y/o amenazas que puedan afectarle;conocer sus fortalezas y debilidades, y poder actuar en correspondencia. 
Las empresas que mejor asimilen las informaciones disponibles, tanto en su ambiente interno como externo, tendrán mayores oportunidades de supervivencia y niveles de competitividad más elevados. Serán capaces de generar mayores beneficios y de contribuir de una mejor forma al desarrollo la región en la que llevan a cabo su actividad. En consecuencia, las organizaciones, sin importar sus dimensiones ni el sector al que pertenezcan, necesitan utilizar mecanismos que, de una forma u otra, permitan recolectar, analizar y utilizar la información disponible a su alrededor para conocer su ambiente, y aprender de él.

A pesar de que no siempre sucede, la información debe ser tratada con la misma seriedad y cuidado que cualquier otro recurso estratégico, porque en caso contrario se pierde la ventaja competitiva, pero también se puede poner en riesgo la propia supervivencia del proyecto empresarial (Starec, 2005: 61).

La información a la que debe hacer frente la empresa es tipologías muy diversas. Pablos Heredero et al.(2012: 28) proponen una clasificación que, a grandes rasgos, intenta resumirla en:

-Información interna/externa. La principal diferencia entre ambas es que mientras la información externa es la procedente de una fuente ajena a la empresa; la información interna es la generada y asimilada como conocimiento dentro de la organización.

-Información de gobierno/consumo. La información de consumo refleja la situación de laempresa; mientras que la de gobierno establece, a modo de plan de acción, la información a futuro: metas, objetivos, etc. que se deben cumplir para mejorar dicha situación.

-Información primaria/secundaria. La información primaria es la obtenida directamente de la fuente principal; la secundaria, por su parte, ha sido tratada respecto a la fuente primaria.

Información contable. Es la relativa a la situación económica de la organización.

-Información administrativa. Trata sobre los aspectos más relevantes de la información contable. Se presenta a los altos cargos de la organización, a modo de resumen ejecutivo.

El volumen de datos e información que las empresas tienen a su disposición hace que en muchas ocasiones los directivos se vean desbordados y se agobien. La información errónea o inadecuada fluye con la misma facilidad que la información correcta o pertinente, sin que sea posible identificarla; con la consecuente pérdida de oportunidades y/o fallos en las decisiones. La sobreabundancia de información es un hecho que según Barboza (2008: 27) afecta a los decisores en el momento de saber en qué información confiar y que según Santos (2000: 205) hace que la gran mayoría de los ejecutivos tenga miedo a equivocarse en el proceso de toma de decisiones. Este hecho provoca que muchas veces tomen decisiones basándose en 
sentimientos, intuiciones y/o en un análisis superficial de las perspectivas de mercado; pero que en pocas oportunidades tengan en cuenta las informaciones adecuadas sobre sus negocios.

Por todo lo anterior, las empresas necesitan llevar a cabo un proceso de gestión que permita el procesamiento, organización y almacenamiento de la información y que asegure su posterior recuperación. Al respecto, Bustelo Ruesta y Amarilla Iglesias(2001: 228)ponen de manifiesto estos aspectos en la definición que aportan del término 'gestión de información’ [GI]:

"el conjunto de actividades realizadas con el fin de controlar, almacenar y, posteriormente, recuperar adecuadamente la información producida, recibida o retenida por cualquier organización en el desarrollo de sus actividades[...]”.

La gestión de información es, por tanto, un proceso intrínseco de las empresas, que puede desarrollarse de forma más o menos consciente y por tanto, con diferentes niveles de eficiencia y efectividad. En la medida que se gestione este recurso de forma eficaz, se podrán explotar las ventajas derivadas del uso de la información y disminuir los costes de su adquisición y tratamiento; mientras que, en paralelo, se asegure su disponibilidad. Escorsa Castells y Maspons Boch(2001: 8) declaran que:

"Una empresa que tenga acceso a información valiosa para su competitividad, en una forma oportuna y adecuada, que cuente con una cultura en la que se promueva la discusión y dondelos miembros clave de la empresa mantengan contacto con fuentes externas de información, ymás importante aún, que pueda transformar esta información en un producto "inteligente", es decir, que proporcione resultados de alto valor estratégico, verá incrementada significativamente sus posibilidades para obtener innovaciones exitosas”.

\subsubsection{LA INFORMACIÓN COMO FUENTE DE CONOCIMIENTO}

Son muchos los autores que han tratado de explicar la relación existente entre la información y el conocimiento, tal y como plantean Fernández y Ponjuán(2008: 2-3). Ambos conceptos están estrechamente relacionados entre sí, pero no son semejantes.El conocimiento es una entidad de trabajo intelectual superior a la información, puesto que es el resultado de la información mentalmente procesada. Este nexo es la clave para entender la incidencia de ambos recursos en la organización como un todo.

La existencia de un importante stock de conocimientos en el interior de las empresas, susceptible a múltiples usos ha propiciado la necesidad de detectar y explotar las ideas valiosas, independientemente de dónde surjan (Jong et al., 2008). Para ello, deben analizarse las fuentes de conocimiento tanto internas (de la propia 
organización) como externas (no pertenecen a la organización). Un hecho que contribuye a poder examinar la situación de las empresas a la luz de los conocimientos disponibles, lo que permite anticiparse a la competencia, identificar debilidades, adaptar las estrategias a medio y largo plazo, etc., con el objetivo de obtener mayores oportunidades de competitividad, y en consecuencia, de supervivencia.

En el caso de las ETBs, dado el uso intensivo que hacen del conocimiento, requieren gestionar tanto los que están disponibles como aquellos que generan en su quehacer diario. En este sentido, Davenport y Prusak(2001: 60)definen el mecanismo de conocimiento dentro de las organizaciones como un proceso dinámico que puede dividirse en tres etapas:

-Generación del conocimiento: ocurre cuando se conciben nuevas ideas, procesos, etc.

-Codificación del conocimiento: se produce cuando la generación puede materializarse.

-Transferencia de conocimiento: es el final del proceso y tiene lugar cuando se aplica y/ o desarrolla el conocimiento para su explotación.

Este último paso permite el intercambio de experiencias, lo que favorece los flujos de información y conocimiento entre la comunidad científica y empresarial. Todo ello se traduce en el desarrollo de nuevos y mejorados proyectos de innovaciónque contribuye a que las empresas se desarrollen y ocupen un sitio en el mercado.

\subsubsection{LA VIGILANCIA TECNOLOGICA / INTELIGENCIA COMPETITIVA COMO HERRAMIENTAS PARA LA GESTIÓN DE LA INFORMACIÓN EXTERNA}

Las empresas necesitan mantenerse informadas de lo que acontece a su alrededor, de forma tal que los cambios del entorno no le pasen desapercibidos y tengan la posibilidad de anticiparse a ellos. En este sentido, Palop y Vicente(1999) reconocen que, por lo general, las empresas suelen seguir la evolución de su entorno de un modo poco formal y organizado, y que la mayoría de ellas practican con mayor o menor rigor alguna forma no consciente de vigilancia tecnológica [VT].

Estos autores definen la vigilancia tecnológica como:

"El esfuerzo sistemático y organizado por la empresa de observación, captación, análisis, difusión precisa y recuperación de información sobre los hechos del entorno económico, tecnológico, social o comercial, relevantes para la misma por poder implicar una oportunidad u amenaza para esta. Requiere una actitud de atención o alerta individual” (Palop y Vicente, 1999: 22). 
La VT busca analizar el comportamiento de los competidores, el mercado, los productos existentes y las nuevas tendencias; es decir, intenta identificar los cambios que se producen en el entorno y que pueden afectar de una forma u otra a la organización. Todo ello a través de la explotación de las fuentes de información disponibles, así como la participación en ferias, eventos y otras actividades comerciales que les permitan obtener un conocimiento del entorno en el que desarrolla su actividad y por ende, adquirir ventajas competitivas.

Muchas veces la VT se asocia a la inteligencia competitiva [IC] y se consideran como sinónimos (Escorsa Castells, 2007: 12). Sin embargo, siguiendo otros enfoques la Vigilancia se incluye dentro de la Inteligencia, considerando a esta última como una evolución la primera(BAI, [s.f]: 16; Rey Vázquez, 2009: 9). En esta línea, se plantea que a diferencia de la VT, la IC "no es solo observación sino una práctica ofensiva y defensiva de la información. Es una herramienta que conecta el saber de la empresa con la acción”(Baumard citado por Escorsa Castells, 2007: 13).

Para Rey Vázquez (2009), la VT se enfoca en el seguimiento de la evolución de la tecnología y de sus implicaciones. Mientras que la IC presta atención a otros factores de la competitividad: competidores actuales y potenciales, clientes, proveedores, entorno normativo, etc., y sus repercusiones en la empresa.

Según Gibbons y Prescott (citados por Escorsa Castells, 2007: 11)la IC es: "el proceso de obtención, análisis, interpretación y difusión de información de valor estratégico sobre la industria y los competidores, que se transmite a los responsables de la toma de decisiones en el momento oportuno". Al mismo tiempo, que Mier (2003) la considera como una función utilizada en las organizaciones con el propósito de que los directivos cuenten con la información estratégica, que les permita tomar acciones efectivas antes de que ocurran los eventos.

Ambas herramientas resultan, por tanto, de vital importancia para el seguimiento de la información externa en la empresa, implican una gestión estratégica de la información que sirve de apoyo a los procesos de toma de decisiones y permiten generar ventajas con respecto a la competencia. De forma general, pueden reportarle notables beneficios, tal y como señalan Medina Vásquez y Ortegón(2006):

-Anticipar cambios: alertan sobre las amenazas y oportunidades provenientes del sector o posibles nuevos nichos de mercado, lo que permite que la organización pueda responder con eficacia.

Reducir riesgos: al detectar competidores o productos entrantes o sustitutos, es posible identificar aquellos que puedan suponer una amenaza para la empresa.

-Clarificar opciones: ayudan a identificar las estrategias y mejores prácticas de $\mathrm{I}+\mathrm{D}+\mathrm{i}$ que puedan llevarse a cabo. 
-Cooperar de forma efectiva al identificar nuevos socios: al establecer su capacidad, idoneidad de trabajo e identificar relaciones Universidad-Empresa, Empresa-Empresa, etc es posible establecer sinergias ventajosas para ambos.

En el caso español, se reconoce la importancia de la VT/IC para la captura, análisis, difusión y explotación de las informaciones científicas o técnicas útiles a la organización, de forma sistemática; pero en la práctica no siempre se aplica. Es por ello, que la Asociación Española de Normalización y Certificación (AENOR) ha elaborado la norma UNE 166006:2006 EX con el propósito de pautar el ejercicio de la actividad de VT dentro de los sistemas de gestión de la I+D+i; en lo que podría considerarse como un punto de partida para la implementación de estos sistemas en las organizaciones, especialmente en las que cuentan con un alto grado de innovación.

La construcción y desarrollo de programas de VT/IC, sin embargo, requiere recursos económicos, tecnológicos y personales que no siempre están disponibles. Por estas razones, su implementación constituye un reto de mayor envergadura para las empresas pequeñas, fundamentalmente aquellas que inician su andadura empresarial; pero que dadas las ventajas que podría generar, no debe ser desdeñado.

\subsection{IMPACTO DE LA INFORMACIÓN EN LAS EBTs}

La dinámica del mercado y los frecuentes cambios tecnológicos que se experimentan en el entorno de las EBTs, hacen que el establecimiento de ventajas competitivas sea mucho más complicado y que estas sean menos duraderas, lo que implica que deban ser constantemente retroalimentadas. En este contexto, la información y el conocimiento se convierten en recursos indispensables para dichas organizaciones. Ambos juegan un papel fundamental en los procesos de innovación y son claves en la competitividad de las empresas(Muñoz Cañavate, 2009: 121; Tanev y Bailetti, 2008: 786).

Todas las actividades que se ejecutan en las empresas, desde la planificación hasta la ejecución de sus procesos, incluida la toma de decisiones están apoyadas por la información y el conocimiento. Sin embargo, en el entorno actual la información alcanza grandes volúmenes; por lo que se hace cada más difícil acceder a la que realmente se necesita. Se requiere un esfuerzo extra en materia de gestión para recolectar, analizar y utilizar aquella que verdaderamente repercuta en el desarrollo de la organización, apoye la innovación y genere ventajas competitivas. Pero, según plantean Saénz de Lacuesta y Bilbao (2004: 168), pocas empresas -y menos aún las PYMEs- consideran la posesión de información estratégica como una inversión de futuro. Incluso, en el caso de que la consideren como tal, no les es fácil decidir qué parte de la información accesible es válida.

En esta misma línea, la falta de reconocimiento del papel de la información en la pequeña empresa, en especial la EBTs, implica que no se gestione adecuadamente las informaciones para hacer frente a los cambios del entorno y a 
los procesos innovadores, lo que para Cubillo (citado por Gomes y Rocha, 2011: 36) contribuye a la alta tasa de mortalidad prematura de este tipo de empresas. Muy pocas son conscientes de la necesidad de desarrollar una adecuada gestión de la información, por lo que muchas veces caen en el error de no reconocer la información disponible en la propia organización por estar fragmentada y dispersa.

Como se ha comentado con anterioridad, las EBTs son intensivas en conocimiento e innovación. La I+D+i que llevan a cabo requiere de información, que convierten en conocimiento y que da lugar a nuevas ideas innovadoras. De ahí que, la comercialización de la información en forma de producto/servicio les proporcione un beneficio económico mayor, cuanta mayor sea la investigación y la innovación que realicen; lo que se revierte en un aumento de la capacidad de inversión para poder investigar. Un hecho especialmente evidente en este tipo de empresas, dados los escasos recursos financieros con que cuentan al comienzo de su actividad y la importancia que tiene la investigación para su desarrollo tecnológico.

Los emprendedores cuentan con una idea, un producto/servicio fruto de la utilización de los recursos existentes de forma creativa e innovadora. Sin embargo, para alcanzar el éxito no pueden limitarse a explotar las capacidades y habilidades con que cuentan, sino que deben explorar su ambiente e identificar las oportunidades. Necesitan desarrollar productos/servicios diferenciadores que les permitan hacer frente a la competencia y ocupar un espacio en el mercado. Pero, este objetivo solo puede lograrse a través del análisis de la información que proporciona el entorno, para lo cual la VT/IC resultan herramientas estratégicas.

Según Merino Moreno (2011: 859) las EBTs necesitan de la VT o de la IC, en dependencia de la fase en la que se encuentre su proyecto emprendedor. En las primeras etapas suelen conceder un papel más relevante a la Vigilancia, por el marcado interés que estas empresas presentan en lo tecnológico y dada su necesidad de confirmar la factibilidad del desarrollo y/o la posibilidad de utilizar combinaciones de soluciones ya existentes que constituyan una oportunidad de producto/servicio renovado. Sin embargo, según se avanza en el proceso de desarrollo empresarial, aparece un momento crítico donde se cambian los papeles protagónicos desde el plano tecnológico al del mercado y la labor de Inteligencia cobra fuerza como eje de referencia para las estrategias de comercialización, es decir, para hacer rentable el esfuerzo, el objetivo básico para cualquier ejercicio empresarial.

En ocasiones, las opciones disponibles dependen de cuán temprano la organización identifique los problemas, mientras que la alternativa más apropiada dependerá de saber reconocer las posibles consecuencias. La utilización de la información contextualizada por la IC permite que las organizaciones comprendan la rapidez de los cambios del entorno, al mismo tiempo que ayuda a reducir la incertidumbre y el riesgo en el proceso de toma de decisiones. No obstante, tal y como señalan García Alcina y Ortoll Espinet(2012: 117) "la inteligencia no tiene valor por sí misma, sino que el valor se crea como resultado de usar la inteligencia, 
diseñando acciones basadas en ella”, es decir, no es el producto de inteligencia sino la decisión que toman los ejecutivos la que tendrá impacto en el resultado final.

A grandes rasgos y aun cuando no siempre se reconoce, la información es el vehículo más apropiado para optimizar los esfuerzos de los emprendedores y obtener mejoras significativas en los procesos de la organización que repercutan en el desarrollo de la misma. Aporta beneficios como:

-Identificar los desarrollos realizados por terceras entidades que estén relacionados con la actividad de la empresa.

-Detectar nuevas necesidades de los clientes y oportunidades de negocio. -Potenciar los flujos de información para agilizar los procesos organizacionales.

-Ahorrar tiempo en la recuperación de la información ya disponible en la empresa.

-Evitar el trabajo duplicado.

-Facilitar la formación de las nuevas incorporaciones a las empresas.

-Establecer una metodología a la que se debe acudir como aprendizaje o como punto de resolución de conflictos.

Para Gomes y Rocha (2011) la simplicidad de la estructura administrativa de las micro y pequeñas empresas puede facilitar el flujo de la información y la comunicación interna. Sin embargo, declaran que la excesiva informalidad y la subjetividad en estos procesos puede comprometer el uso posterior de las informaciones que, en su gran mayoría, quedan almacenadas en la 'cabeza del directivo principal'. De cara a un proceso de gestión de la información, se hace necesario por tanto, reconfigurar el funcionamiento de las organizaciones y del personal que participa de sus procesos, de forma tal que se establezcan las condiciones idóneas para la creación y el intercambio de información y conocimiento entre los trabajadores.

Otro de los aspectos que demuestra la importancia de la información en la empresa, son los documentos que esta genera como reflejo de su actividad y que son trasmisores de la información que explicita su conocimiento organizacional. Son muchos los tipos de documentos que pueden estar presentes en una empresa, sean o no generados por ella misma. Pero, sin duda, es necesario llevar a cabo una gestión de los mismos que asegure su procesamiento y almacenamiento, como garantías para su posterior recuperación y uso.

Por otra parte, la variedad de fuentes que están a disposición de los emprendedores y que proporcionan información especializada constituye una fortaleza para las organizaciones, si estas son capaces de explotarlas en toda su plenitud. Sin embargo, contrario a esta realidad, Muñoz Cañavate (2012: 38) plantea que diversos estudios realizados en España destacan que una de las quejas que tienen los empresarios es la falta de información. Un hecho ante el cual dicha 
autora se cuestiona si no estará más relacionado con la formación del empresariado español, más preocupado por la gestión del día a día, donde aparentemente no intervienen aspectos como la gestión de la información y la gestión del conocimiento.

Las EBTs, y en general cualquier organización, requieren de informaciones muy diversas para el desempeño de sus funciones. Por un lado, necesitan de fuentes que les permitan conocer el entorno, tales como estudios de mercado, estudios sociológicos, estadísticas, encuestas de opinión, etc. medios de comunicación, redes sociales, etc. Mientras que por el otro, requieren de fuentes tales como revistas especializadas, bases de datos científicas, patentes, etc. que les ayude a sustentar y desarrollar sus procesos $\mathrm{I}+\mathrm{D}+\mathrm{i}$. Al mismo tiempo que necesitan de contactos personales con clientes, consultores, proveedores, etc. y del intercambio de información producido en las ferias, congresos, seminarios y conferencias para obtener información de utilidad para la empresa. Al respecto, CETISME (2002: 81) señala que la integración de diferentes fuentes de información parece ser la solución más efectiva para las pequeñas empresas, tanto desde un punto de vista económico como cuantitativo. Las fuentes tradicionales pueden complementarse con las personales en dependencia del tipo de decisión que se vaya a apoyar.

El caso de las patentes como fuente de información, se reviste de una especial relevancia para las PYMEs. La información que aportan resulta crucial para la previsión tecnológica y para proyectar los consecuentes cambios del mercado. Sin embargo, a pesar de su valor, su utilización no está muy extendida, lo que conlleva a gastos innecesarios de tiempo y recursos por la duplicidad de trabajos.

Los elementos anteriores demuestran cómo la información incide en la actividad de las EBTs y evidencian la necesidad de su gestión; a pesar de que la gestión de la información, en función de la innovación y la competitividad, se suele asociar más a las grandes empresas, en detrimento de las pequeñas. Al respecto, Tanev (2007) reconoce que la relación entre la gestión de información, en especial la correspondiente a la VT/IC, y los resultados de la innovación de las pequeñas empresas es importante, pero se sabe poco sobre la misma. No obstante, dados los beneficios que las PYMEs podrían obtener con su implementación, debería ser una cuestión a tener en cuenta.

Los emprendedores suelen empezar sus proyectos en solitario o con un reducido número de colaboradores, lo que hace que la gestión de la información en las EBTs sea aún más complicada y costosa. Por una parte, cuentan con pocos conocimientos para llevarla a cabo, lo que afecta su capacidad para organizar, formalizar y descifrar el valor estratégico de la información (Gomes y Rocha, 2011: 36); y por la otra carecen del tiempo necesario para ello. Al tiempo que tampoco se encuentran en condiciones para destinar recursos que permitan el desarrollo de esta actividad internamente. 
Para dar solución a este problema y que las EBTs puedan contar con los beneficios de esta actividad, García Alsina y Ortoll Espinet(2012: 70)proponen externalizar algunos de sus servicios, concretamente los de IC, a través de consultoras especializadas. Una fórmula que, aunque podría implicar la salida de la empresa de la información confidencial, es una vía rápida y eficaz para obtener información relevante. Otra alternativa, que no tiene por qué ser excluyente, podría ser que los parques científicos y tecnológicos, o en su defecto, los viveros de empresas donde se insertan la mayoría de estas organizaciones, se involucren en el proceso de gestión de información y ofrezcan servicios de este tipo a sus empresas incubadas.

\subsubsection{LOS PARQUES CIENTÍFICOS Y TECNOLÓGICOS COMO MEDIADORES EN EL USO DE LA INFORMACIÓN}

El rol de los parques científicos y tecnológicos y de los viveros de empresa va más allá de funcionar como inquilinos o asesores de las EBTs que acogen como parte de los programas de incubación que desarrollan. Por un lado, pueden facilitar la creación de una red de contactos con otras empresas dentro del vivero y con agentes externos: profesores universitarios, personal de la industria, consultores, etc. que proporcione un flujo continuado y permanente de clientes y proveedores (Gómez, 2002: 4). Mientras que por el otro, pueden promover la transferencia de conocimientos y el intercambio de información entre las empresas. Al respecto, Iacono y Nagano(2014 p. 298) reconocen que el establecimiento de una amplia red de contactos y socios, promueve el acceso a la información, el conocimiento y el mercado, lo que es de gran importancia para estas empresas.

La preocupación por generar, difundir y compartir la información y el conocimiento en los parques contribuye a que las empresas incubadas alcancen las condiciones requeridas para convertirse, en un período relativamente corto, en empresas capaces de competir con otras en la misma industria, sector o actividad, independientemente de la región o país de origen (Raupp y Beuren, 2007: 56). En este sentido, es válido señalar que la eficacia de los servicios prestados a los empresarios incubados se considera un factor decisivo para el éxito del vivero ya que se puede entender como la formación de empresas más duraderas y exitosas (Xavier, Martins y Lima, 2008: 435-6).

Como impulsores de los nuevos proyectos empresariales, estas instituciones podrían establecer acciones de formación que ayuden a los emprendedores a identificar la importancia de la información en sus procesos y a aprender a gestionar este recurso, de forma que se revierta en beneficio de la organización. Igualmente podrían profundizar en los conocimientos de los emprendedores en materia de fuentes de información y de las herramientas disponibles para su búsqueda y recuperación, así como para el análisis de la información. 
Los parques buscan el fortalecimiento de las habilidades de las empresas incubadas y el enriquecimiento de su base de conocimientos. De ahí que estén en las mejores condiciones para promover la creación de oportunidades y canales de comunicación que ayuden a producir y transferir información a las empresas involucradas en el proyecto. Pueden generar espacios para que dichas empresas compartan entre sí sus propias experiencias y conocimientos, generando así una sinergia que contribuya a la creatividad y al desarrollo de competencias básicas para alcanzar el éxito empresarial. Al respecto, Kase y Zupan (citados por Adlesic y Slavec, 2012: 207) señalan que en las empresas intensivas en conocimiento la creación de un ambiente informacional que ayude a los empleados a resolver problemas cada vez más complejos $\mathrm{y}$, a menudo ambiguos, contribuye de manera significativa al rendimiento.

Estas instituciones podrían actuar como núcleos o centros de información del cual se sirvan las empresas incubadas en los procesos de obtención y análisis de la información, identificación de empresas y/o grupos de investigación con actividades relacionadas, conocimiento del mercado, etc. Para ello deberían ofrecer, teniendo en cuenta las necesidades de los emprendedores y de forma sistemática, servicios que proporcionen información relevante para su actividad y que los empresarios puedan trasformar en conocimiento e innovación que les permita lograr mayores ventajas competitivas.

Según Barreto (1999: 2), los parques deben asumir su rol como unidades de transferencia de conocimiento, es decir, unidades que formalizan un proceso de transferencia que permite el flujo completo de conocimiento, que va desde la asimilación de la información por parte del receptor hasta su transformación en nuevos conocimientos. En este sentido, contar con el apoyo y/o la asesoría de profesionales de la información y la documentación que les provean de los conocimientos necesarios sobre el tema, podría ser de gran utilidad.

\section{CONCLUSIONES}

Los documentos relevantes localizados durante la revisión bibliográficahan permitido elaborar una primera aproximación al papel de la información en las empresas de base tecnológica, desde la perspectiva de la literatura profesional. Los planteamientos realizados por los autores consultados evidencian el valor de la información como pilar de los procesos organizacionales, en especial de los de I+D+i y los de toma de decisiones; así como la necesidad de una gestión estratégica de este recurso que permita generar ventajas competitivas y contribuya al posicionamiento de las EBTs en su mercado.

Los estudios prácticos más representativos han sido el de Gomes y Rocha (2011) y el de Periotto (2010) en relación con la gestión de la información, y los Tanev(2007; Tanev y Bailetti, 2008) en cuanto a la inteligencia competitiva. En 
este último aspecto, desde un análisis más teórico, ha sido igualmente significativo el artículoelaborado por Merino Moreno (2011).

En cuanto al estado del arte, puede concluirse que las EBTs manejan información de forma permanente, ya sea consciente o inconscientemente, y con independencia de la etapa de desarrollo organizacional en la que se encuentren. Pero, no hacen un uso intensivo de la misma como fuente de conocimiento y por ende, no explotan todo su potencial en beneficio de los procesos de innovación intrínsecos a estas empresas.

Estas organizaciones reconocen sus carencias en materia de gestión financiera, pero no son capaces de identificarlas en relación con la gestión de la información. Muy pocas son conscientes de la necesidad de llevar a cabo una adecuada gestión de este recurso que les permita hacer frente a los cambios del entorno y a los procesos innovadores. A lo que se añade que, los emprendedores no suelen poseer los conocimientos necesarios ni el tiempo requerido para realizarlo.

La preocupación de los parques científicos y tecnológicos por generar, difundir y compartir la información y el conocimiento contribuye a que las EBTs incubadas alcancen las condiciones requeridas para competir en el mercado, en un período relativamente corto. Dichas instituciones podrían actuar como núcleos o centros de información del cual se sirvan las EBTs y ofrecerles servicios que les proporcionen información que puedan transformar en conocimiento e innovación. En este contexto, los profesionales de la información y la documentación pueden ser un importante activo a tener en cuenta, pues disponende las competencias necesarias para implementarsoluciones que respondan a las necesidades informacionales de estas empresas.

\section{BIBLIOGRAFÍA}

ADLESIC, Renata V. y SLAVEC, Alenka (2012), "Social capital and business incubators performance: testing the structural model ". Economic and Business Review for Central and South - Eastern Europe [en línea], vol. 14, no. 3, pp. 20122. <http://search.proquest.com/docview/1315885605>. [Consulta: 25 ago. 2014].

APORTELA RODRÍGUEZ, Ivett. (2014) "Information and Knowledge in Spanish Science and Technology Parks", en VIVAS,C., y P. SEQUEIRA Proceedings of the 15th European Conference on Knowledge Management (Santarem, Portugal, Sep 2014) [en línea], Kidmore End, Academic Conferences International Limited, 2014 , vol. 3, p. 1099-1107. $<$ http://search.proquest.com/docview/1673123787?accountid=14501>.

[Consulta: 15 ene. 2015].

BAI, ([s.f]). Modelos de vigilancia tecnológica e inteligencia competitiva [en línea]. Bizkaia: BAI agencia de innovación. $<$ www.centrodecompetitividaddelmaule.cl/files/MODELOS-DE-IC-EN-ELMUNDO.pdf $>$. [Consulta: 18 jul. 2014]. 
BARBOZA, Elder L. (2008) Gestão estratégica da informação e a inteligência competitiva: estudo exploratório para a implantação no Conselho de Consumidores da Enersul - CONCEN [en línea]. Orlando de Almeida Filho, dir. . Trabajo fin de curso de bachiller. Instituto de Ensino Superior da FUNLEC - IESF, Campo Grande [Brasil]. $<$ http://bibliotecariosms.files.wordpress.com/2009/07/elder-lopes-barbozagestao-estrategica-da-informacao-e-a-inteligencia-competitiva.pdf $>$.

[Consulta:23 ago. 2012].

BARRETO, Aldo A. (1999), "A oferta e a demanda da informação: condições técnicas, econômicas e políticas". Ciência da Informação [en línea], vol. 28, no. 2. <www.scielo.br/pdf/ci/v28n2/28n2a09.pdf>. [Consulta: 28 ago. 2012].

BUSTELO RUESTA, Carlota y AMARILLA IGLESIAS, Raquel (2001), "Gestión del conocimiento y gestión de la información". PH: Boletín del Instituto Andaluz de Patrimonio Histórico [en línea], mar. 2001, vol. VIII, no. 34, pp. 226-30.<www.intercontact.com.ar/comunidad/archivos/ Gestion_del_Conocimiento-BusteloRuesta-AmarillaIglesias.pdf>. [Consulta: 09 abr. 2015].

CASTILLO HERMOSA, Jaime y DÍEZ FUENTE, Carlos (2006), "Infraestructuras de apoyo a la innovación científica y tecnológica: elementos clave de competitividad industrial". Ekonomiaz [en línea], sept-dic. 2006, no. 63, pp. 128-51. <www1.euskadi.net/ekonomiaz/downloadPDF.apl?REG=821>. [Consulta: 23 ago. 2014].

CETISME (2002). Inteligencia Económica y Tecnológica: Guía para principiantes y profesionales [en línea]. Madrid: Comunidad de Madrid, Dirección General de Investigación. < www.madrimasd.org/informacionidi/biblioteca/publicacion/ doc/15_InteligenciaEconomicaTecnologica.pdf >. [Consulta: 18 jul. 2014].

DAVENPORT, Thomas H. y PRUSAK, Laurence (2001). Conocimiento en acción: Cómo las organizaciones manejan lo que saben. Buenos Aires: Prentice Hall.

ESCORSA CASTELLS, Pere (dir cientif.) (2007). INTEC, la inteligencia competitiva: factor clave para la toma de decisiones estratégicas en las organizaciones [en línea]. Pilar Lázaro, coord ed. Madrid: Comunidad de Madrid, Dirección General de Universidades e Investigación: Fundación madri+d para el Conocimiento, 2007, vol. 35. < www.madrimasd.org/informacionidi/biblioteca/ publicacion/doc/35_intec.pdf>. [Consulta: 18 jul. 2014].

ESCORSA CASTELLS, Pere y MASPONS BOCH, Ramon (2001). De la Vigilancia Tecnológica a la inteligencia Competitiva. Madrid: Pearson Educación.

FELIÚ REY, Jorge (2011), "Empresas de base tecnológica o spin off académica: aproximación a su concepto, antecedentes y algunas consideraciones de derecho privado". Derecho de los Negocios, Dic. 2011, no. 255, pp. 7-22.

FERNÁNDEZ VALDÉS, María M. y PONJUÁN DANTE, Gloria (2008), "Análisis conceptual de las principales interacciones entre la gestión de 
información, la gestión documental y la gestión del conocimiento". ACIMED [en línea], vol. 18, no. 1. <http://scielo.sld.cu/pdf/aci/v18n1/aci07708.pdf>. [Consulta: 25 ago. 2014].

GARCÍA ALCINA, Monserrat y ORTOLL ESPINET, Eva (2012). La inteligencia competitiva: evolución histórica y fundamentos teóricos. Gijón: TREA.

GOMES, Suely y ROCHA, Jaqueline (2011), "Gestão da informação: o caso das empresas participantes do programa de incubação de empresas da Universidade Federal de Goiás". Palabra Clave (La Plata) [en línea], oct. 2011, vol. 1, no. 1, pp. 21-39. <www.memoria.fahce.unlp.edu.ar/art_revistas/pr.4901/ pr.4901.pdf>. [Consulta: 10 ene. 2014].

GÓMEZ, Liyis (2002), "Evaluación del impacto de las incubadoras de empresas: estudios realizados". Pensamiento \& Gestión: Revista de la División de Ciencias Administrativas de la Universidad del Norte [en línea], Dic. 2002, no. 13, pp. 1-22. <http://maaz.ihmc.us/rid=1177390614203_1717588426 1282/EvaluaciondelImpactodelasIncubadorasdeEmpresasGOMEZ_2002.pdf>. [Consulta: 27 ago. 2012].

IACONO, Antonio y NAGANO, Marcelo S. (2014), "Gestão da inovação em empresas nascentes de base tecnológica: evidências em uma incubadora de empresas no Brasil". Interciencia [en línea], May 2014, vol. 39, no. 5, pp. 296306. <http://search.proquest.com/docview/1535664207?accountid=14501>. [Consulta: 7 jul. 2014].

JONG, Jeroen P. J., et al. (2008). Policies for open innovation: Theory, framework and cases [en línea]. Helsinki: VISION Era-Net, Jul. 2008. $<$ http://www.praxis.ee/wp-content/uploads/2014/03/2008-Policies-for-openinnovation.pdf $>$. [Consulta: 18 jul. 2014].

LÖFSTEN, Hans (2014), "Information Structures and Business Performance: Implications for Technology-based Firm's Innovation Performance". Knowledge \& Process Management [en línea], Oct, vol. 21, no. 4, pp. 246-59. DOI: 10.1002/kpm.1446. [Consulta: 15 ene. 2015].

MEDINA VÁSQUEZ, Javier y ORTEGÓN, Edgar (2006). Manual de prospectiva y decisión estratégica: bases teóricas e instrumentos para América Latina y el Caribe [en línea]. Santiago de Chile: CEPAL, Instituto Latinoamericano y del Caribe de Planificación Económica y Social. $<$ http://www.cepal.org/ilpes/publicaciones/xml/3/27693/manual51.pdf $>$. [Consulta: 14 ago. 2014].

MERINO MORENO, Carlos (2011), "Inteligencia competitiva en el contexto de las nuevas empresas de base tecnológica". Ide@s CONCYTEG [en línea], Jul 2011, vol. 6, no. 73, pp. 845-62. <http://octi.guanajuato.gob.mx/octigto/ formularios/ideasConcyteg/Archivos/73042011_MERINO.pdf >. [Consulta: 18 jul. 2014]. 
MUÑOZ CAÑAVATE, Antonio (2012). Recursos de información para la inteligencia competitiva: Una guia para la toma de decisiones. Gijón: Trea, 2012.

- (2009), "La información para la empresa y el sistema de I+D+i. el caso de la región valenciana". Revista General de Información y Documentación, vol. 19, pp. 121-44.

NETTO, Antonio V. (2006). Gestão das pequenas e médias empresas de base tecnológica. Barueri: Minha; Brasília: Sebrae, 2006.

NIETO, María J. y SANTAMARÍA, Lluis (2010), "Colaboración tecnológica e innovación en las empresas de base tecnológica: implicaciones de las relaciones con universidades y otros socios tecnológicos". Revista Galega de Economía [en línea], vol. 19, pp. 1-15. <http://www.redalyc.org/articulo.oa?id=39115737009>. [Consulta: 22 ago. 2014].

PABLOS HEREDERO, Carmen, et al. (2012). Organización y transformación de los sistemas de información en la empresa. Madrid: ESIC.

PALACIOS, Miguel; VAL, Tíndaro y CASANUEVA, Carlos (coord.) (2005) "Inversión en nuevas empresas de base tecnológica en la Comunidad de Madrid", en Madri+d [en línea]. Madrid: Fundación para el Conocimiento madri+d<www.madrimasd.org/emprendedores/uploads/biblioteca/Inversion_N EBTs.pdf $>$. [Consulta: 18 jul. 2014].

PALOP, Fernando y VICENTE, José M. (dirs.) (1999) "Vigilancia tecnológica e inteligencia competitiva: su potencial para la empresa española", en Navactiva.com [en línea], actualizado feb. 1999. <http://info.uned.es/expertouniversitario-gestion-I D/bibliografia/VigilanciaTecnologica_inteligencia \%20competitiva.pdf $>$. [Consulta: 18 jul. 2014].

PÉREZ GONZÁLEZ, Daniel y PLACER MARURI, Emilio (2011), "Vigilancia tecnológica en PyMES industriales del metal: conocimiento, aplicación y medición de sus beneficios". El Profesional de la Información [en línea], sep. 2011, vol. 20, no. 5, pp. 495-502. <http://web.b.ebscohost.com/ehost/>. DOI: 10.3145/epi.2011.sep.02. [Consulta: 8 jul. 2014].

PERIOTTO, Caroline. (2010) Análisis e uso da informaçao em pequenas empresas de base tecnologica incubadas no Pólo Tecnológico de São Carlos $S P$ [en línea]. Wanda Ap Machado Hoffman, dir. . Tesis de Maestría. Universidad Federal de São Carlos, 163p. $</$ www.bdtd.ufscar.br/htdocs/tedeSimplificado// tde_busca/arquivo.php?codArquivo=3312>. [Consulta:14 jun 2012].

RAUPP, Fabiano M. y BEUREN, Ilse M. (2007), "Compartilhamento do conhecimento em incubadoras brasileiras associadas à ANPROTEC". Revista de Administração Mackenzie [en línea], 2007, vol. 8, no. 2, pp. 38-58. $<$ http://redalyc.uaemex.mx/src/inicio/ArtPdfRed.jsp?iCve=195415185003>. [Consulta: 26 ago. 2012]. 
Real Academia Española (2001). Diccionario de la lengua española [en línea]. $22^{\mathrm{a}}$ ed. Madrid: RAE. <http://lema.rae.es/drae/>. [Consulta: 25 ago. 2014].

REY VÁZQUEZ, Lara (2009). Informe APEI sobre vigilancia tecnológica [en línea]. Gijón: APEI. <http://eprints.rclis.org/14114/1/INFORME_APEI_ 04.pdf $>$. [Consulta: 19 jul. 2014].

SAÉNZ DE LACUESTA, Sonia y BILBAO, Maitane (2004), "Integración de agentes regionales de innovación y prestación de servicios avanzados de vigilancia tecnológica e inteligencia competitiva para PYMEs: el caso Zaintek". Scire: Representación y organización del conocimiento [en línea], jul.-dic. 2004, vol. 10, no. 2, pp. 167-71. <http://ibersid.eu/ojs/index.php/scire/article/\%20view/1503/1481>. [Consulta: 19 jul. 2014].

SANTOS, Raimundo N. (2000), "Métodos e ferramentas para gestão de inteligência e do conhecimento". Perspectivas em Ciencia da Informaçao [en línea], jul-dic 2000, vol. 5, no. 2, pp. 205-15. $<$ http://portaldeperiodicos.eci.ufmg.br/index.php/pci/article/view/125/322>. [Consulta: 29 jun. 2012].

SOMSUK, Nisakorn; WONGLIMPIYARAT, Jarunee y WONGLIMPIYARAT, Tritos (2012), "Technology business incubators and industrial development: resource-based view". Industrial Management \& Data System [en línea], vol. 112, no. 2, pp. 245-67. DOI: 10.1108/02635571211204281. [Consulta: 8 jul. 2014].

STAREC, Claudio (2005), "A dinâmica da informação: a gestão estratégica da Informação para a tomada de decisão nas organizações" en STAREC, Claudio;GOMES, Elizabeth, y BEZERRA, Jorge Gestão Estratégica da Informação e Inteligência Competitiva. Barra Funda: Saraiva, 2005, pp. 47-64.

TANEV, Stoyan. (2007) "Competitive intelligence information management and innovation in small technology-based companies", en TUCHIN,Valery V. Proceedings of SPIE 6535, Saratov Fall Meeting 2006: Optical Technologies in Biophysics and Medicine VIII (Saratov, Sep. 2006) [en línea], 2007 , vol. 653518, p. 53518-1-53518-17. <http://proceedings.spiedigitallibrary.org/ on 05/09/2014>. DOI10.1117/12.740978. [Consulta: 8 jul. 2014].

TANEV, Stoyan y BAILETTI, Tony (2008), "Competitive intelligence information and innovation in small Canadian firms". European Journal of Marketing [en línea], vol. 42, no. 7-8, pp. 786-803. DOI: 10.1108/03090560810877150. [Consulta: 8 jul. 2014].

XAVIER, Wescley S.; MARTINS, Guilherme S. y LIMA, Afonso A. T. (2008), "Fortalecendo empreendimentos em TI: qual a contribuição das incubadoras?". Journal of Information Systems and Technology Management (Online) [en línea], 2008, vol. 5, no. 3, pp. 433-52. $<$ http://www.revistasusp.sibi.usp.br/scielo.php?script=sci_arttext\&pid=S180717752008000300001\&lng=pt\&nrm=iso>. [Consulta: 25 ago. 2012]. 